\title{
Tailored Nasal Reconstruction after Dog-Bite Injury in a Young Woman
}

\author{
Milena Ferraro ${ }^{1}$ Rosa Alessia Battista ${ }^{1,2, \odot}$ Leone Giordano ${ }^{1}$ Mario Bussi ${ }^{1,2}$
}

\author{
${ }^{1}$ Department of Otolaryngology-Head and Neck Surgery, IRCCS \\ San Raffaele Scientific Institute, Milan, Italy \\ ${ }^{2}$ Vita-Salute San Raffaele University, Milan, Italy
}

Indian J Plast Surg 2020;53:157-158

Due to the prominence in the facial framework, nose is commonly susceptible to accidental injury, with functional and psychological related damage. The aim of nasal reconstruction is the full restoration of the complex nasal architecture, made on concave and convex osteocartilaginous surfaces with cutaneous and mucosal linings.

Optimal treatment is still debated. According to literature, standard criterion for nasal reconstruction is the frontal flap. ${ }^{1}$ Despite its safety and reliability, due to a robust pedicle and large amount of tissue, this option implies a central-facial esthetic defect. Auricular composite grafts can be a good option, taking into account the minor donor site comorbidity and esthetic defect. However, viability remains a concern and affects the maximal graft width, as recommended by Ahuja et al. ${ }^{2}$ Most failures occur in tobacco users or in cardiovascular and diabetic patients where microvascular circulation can be compromised. ${ }^{3} \mathrm{~A}$ tailored therapy has to be planned based on age, sex, and comorbidities.

Herein, we present a 28-year-old female patient, victim of dog bite resulting in avulsion of the right nasal ala with irregular wound $(1.8 \mathrm{~cm} \times 2.0 \mathrm{~cm})$. The defect included several
Address for correspondence Leone Giordano, MD, Department of Otolaryngology-Head and Neck Surgery, IRCCS San Raffaele Scientific Institute, Via Olgettina 60, Milan, 20132, Italy (e-mail: giordano.leone@hsr.it).

nasal subunits (partial nasal tip, medial third of right alar cartilage, and soft triangle) besides the total avulsion of skin, subcutaneous fat, cartilage, and mucosa, falling in IIIB category of modified Lackmann's classification for facial wound. ${ }^{4}$ The avulsed portion was not retrieved. The patient immediately refused the forehead flap reconstruction, scared by predictable poor esthetic results. Considering young age and absence of comorbidities, we opted for an immediate nasal reconstruction with auricular composite graft. ${ }^{5}$ This choice would not compromise an eventually second reconstruction with the gold-standard flap. Furthermore, we fully informed the patient about the possibility of a secondary surgical procedure with frontal flap in case of composite graft failure.

The homolateral helix was chosen as donor site and the graft was demarcated and sculpted based on the size and shape of the nasal defect, to harvest a cutaneous and cartilaginous flap. Donor site's defect was remodeled at the end of the procedure using a superior-based preauricular rotation flap (-Fig. 1).

At 1-year follow-up, nasal ala shape was stable, color was consistent with surrounding tissue, and satisfactory
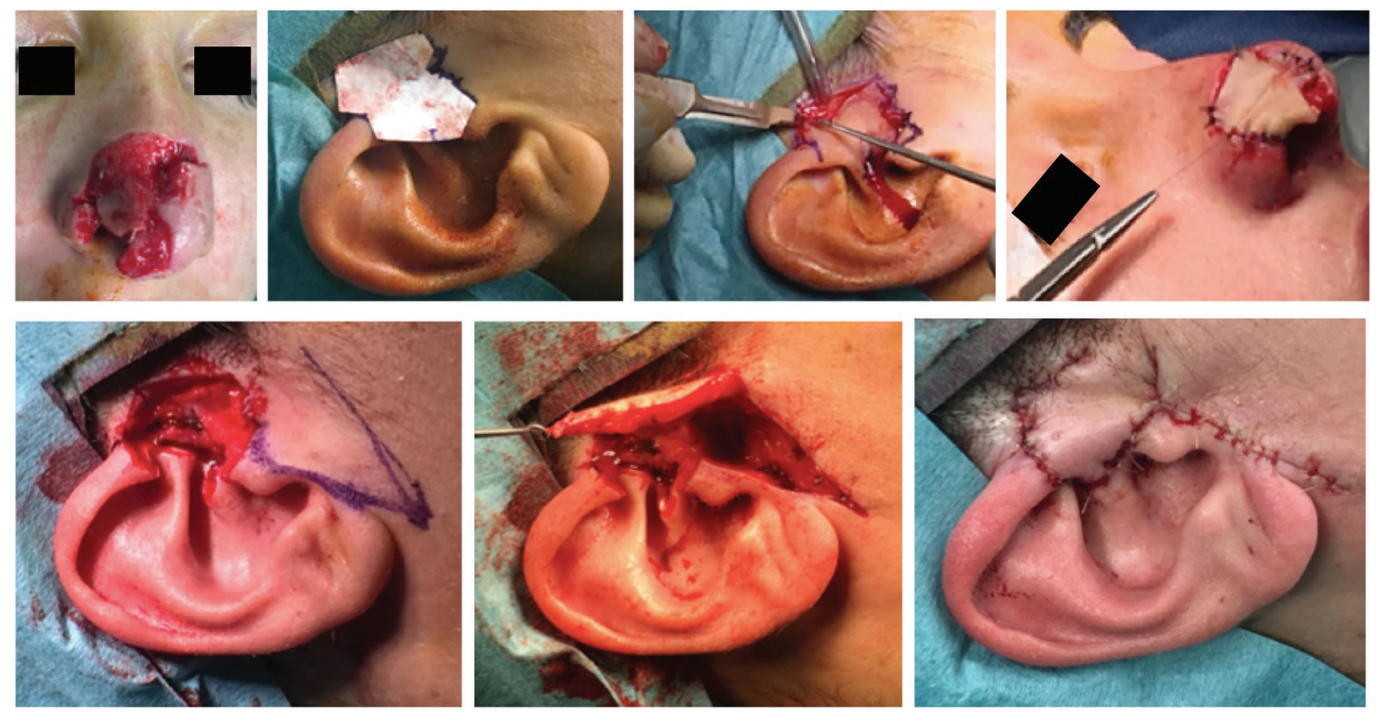

Fig. 1 Surgical steps of the nasal and donor site reconstruction.

published online

April 14, 2020
DOI https://doi.org/

10.1055/s-0040-1709811

ISSN 0970-0358.
C2020 Association of Plastic

Surgeons of India
License terms

() (1) $\ominus \circledast$ 

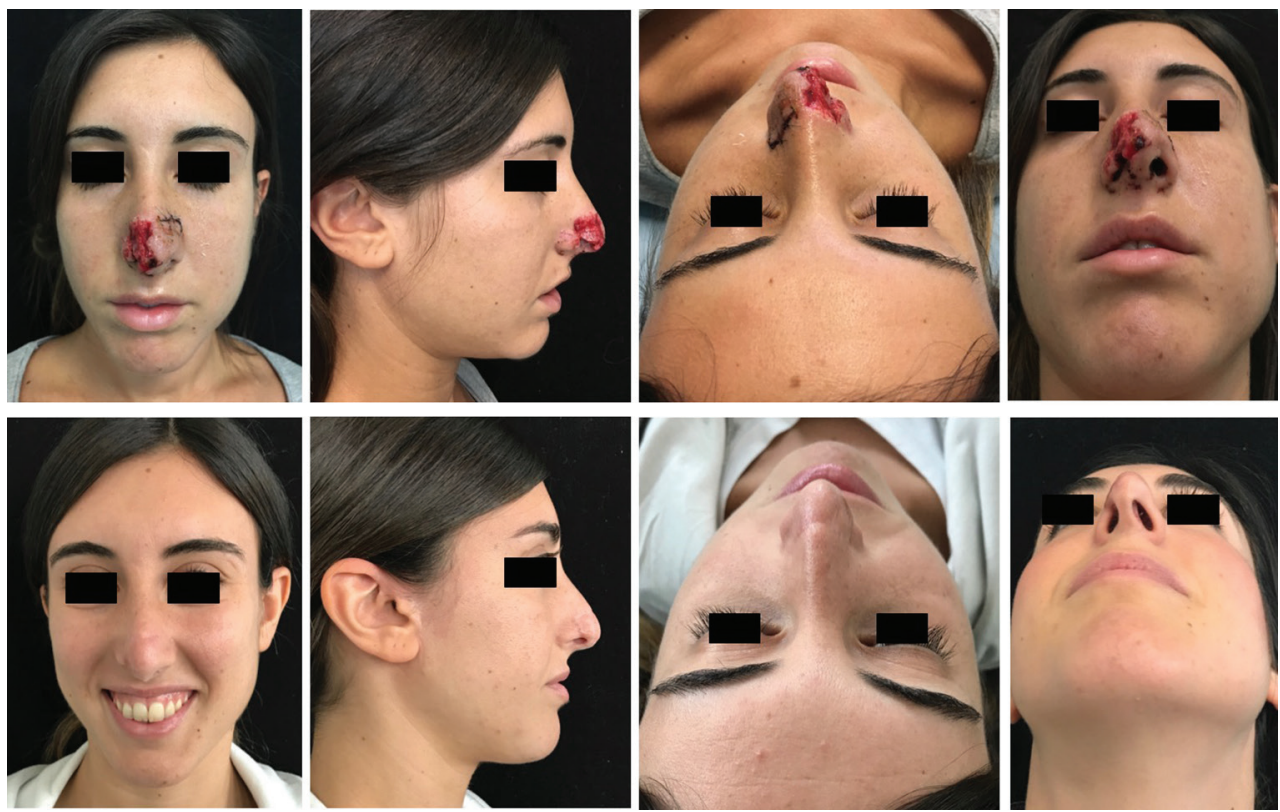

Fig. 2 Pre- and postoperative results comparison at 1-year follow-up.

symmetry was achieved without aesthetic defects in size and framework of the ear ( - Fig. 2).

Auricular composite graft demonstrated to be a safe option to reconstruct nasal ala injuries in young healthy patients when no conditions of reimplantation exist.

\section{Conflict of Interest}

None declared.

\section{References}

1 Shokri T, Kadakia S, Saman M, et al. The paramedian forehead flap for nasal reconstruction: from antiquity to present. J Craniofac Surg 2019;30(2):330-333

2 Ahuja RB, Gupta R, Chatterjee P, Shrivastava P. Securing aesthetic outcomes for composite grafts to alar margin and columellar defects: a long term experience. Indian J Plast Surg 2014;47(3):333-339

3 Woodard CR, Park SS. Reconstruction of nasal defects $1.5 \mathrm{~cm}$ or smaller. Arch Facial Plast Surg 2011;13(2):97-102

4 Chhabra S, Chhabra N, Gaba S. Maxillofacial injuries due to animal bites. J Maxillofac Oral Surg 2015;14(2):142-153

5 Spataro E, Branham GH. Principles of nasal reconstruction. Facial Plast Surg 2017;33(1):9-16 